\title{
Modeling the Non Linear Behavior of a Magnetic Fault Current Limiter
}

\author{
Peter Wilson \\ Department of Electronic and Electrical Engineering, \\ University of Bath, Bath, UK \\ *corresponding author, E-mail: p.r.wilson@bath.ac.uk
}

\begin{abstract}
Fault Current Limiters are used in a wide array of applications from small circuit protection at low power levels to large scale high power applications which require superconductors and complex control circuitry. One advantage of passive fault current limiters (FCL) is the automatic behavior that is dependent on the intrinsic properties of the circuit elements rather than on a complex feedback control scheme making this approach attractive for low cost applications and also where reliability is critical. This paper describes the behavioral modeling of a passive Magnetic FCL and its potential application in practical circuits.
\end{abstract}

\section{Introduction}

The development of Passive Fault Current Limiting devices (FCL) for the protection of electronic systems has been required due to the increase in load growth in conjunction with a requirement for increased reliability. The basic technique uses a magnetic core that has been magnetically offset such that the material is fully saturated. When a winding around this core is connected as an inductor in series with a line, the inductance (and hence the impedance) is low and so the line current is allowed to pass through unhindered. If the current reaches a level high enough to bring the core material out of saturation, then the inductance will increase dramatically and block the current, thus providing current limiting.

A variety of techniques exist to accomplish the protection with the overall requirements for such systems discussed in [1]. The literature has extensive discussion of the range of approaches including Microprocessor control [2], an Electromagnetic method [3], Direct Cooled Tubular Element [4], a tuned LC circuit [5] and numerous papers investigating the use of superconductors including $[6,7,8]$. These techniques all suffer to some extent from being complicated, expensive and difficult to implement. The use of permanent magnets to provide a cheap and simple alternative approach for magnetic offset in inductors has been presented by Mukhopadhyay et al [9], Dawson, Yamada and Iwahara [10], with specific tableau numerical methods introduced by Iwahara and Miyazawa [11]. More recent attempts have been made such as by Rozenshtein et al [15] separating the DC Bias and AC coils to improve the practical realization of the FCL. The permanent magnet approach has potential due to the low cost, simple construction and potentially good transition characteristics to and from the fault region of operation. The challenge for researchers in this field has been trading off the relative simplicity of the design, with the ability to accurately design the non-linear behavior accurately enough, and to be able to construct a practical FCL for a reasonable cost. Hall and Cheer [16] have investigated the use of ceramic based magnets to attempt to reduce the cost and complexity of the design. This is also an example of one approach to modelling these magnetic devices which is based on static finite element analysis of the device and then application of the resulting behavior into a circuit context. The previous work by Mukhopadhyay et al [9] has also modeled these devices using a simple piece wise linear (PWL) approximation of the non-linear behavior of the core material, however the comparison of simulated with measured results has been quantitatively deficient. The authors themselves make no claims in this regard with both the overall current limiting effect subject to large tolerances, and the comparison between measured and simulated curves shows significant differences. For this reason, this paper tackles the problem of accurate prediction of passive FCL behavior from the perspective of detailed nonlinear models of magnetic materials in conjunction with an electrical circuit models.

\section{Modeling Philosophy}

The regions of low and high permeability in a typical magnetic material are illustrated by Figure 1, which shows how the permeability (and hence impedance) increases as the current deviates from its nominal operating condition in the low permeability region. One aspect of this type of approach is that if the Limiter is magnetically offset, then it can only limit the current deviations in one direction. To achieve bi-polar current limiting requires two of these devices, with the second device having the opposite magnetic offset.

It is clear from Figure 1 that if accurate models for the core material behavior could be obtained, then reliable predictions about the performance of the Passive Fault Current Limiter could potentially be achieved. In this paper, classical magnetic material and component modeling techniques previously developed [12] and [14] are applied to provide a much more accurate representation of the passive Fault Current Limiter behavior. The modeling was approached from a mixed-domain point of view, using a JilesAtherton magnetic material model including hysteresis and power loss $[17,18,19,20,21]$. By modeling the hystere- 


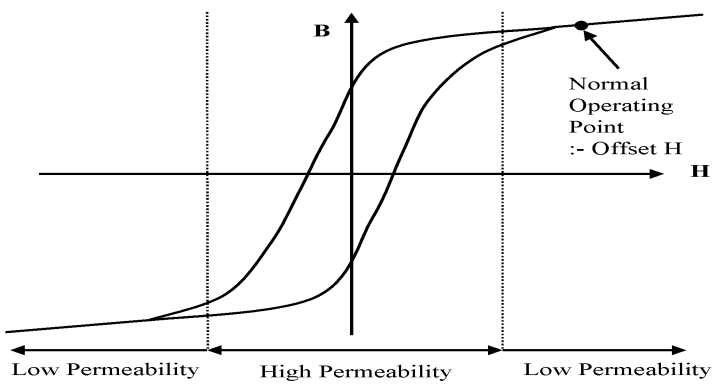

Figure 1: Fault Current Limiter Operating Regions

sis of the core material, the limit and recovery cycle can be predicted more accurately than previously obtained using the simple PWL approach. Measured and Simulated results were obtained for FCL components using an external permanent magnet Aspects of the FCL component are discussed including eddy currents, and the behavior of the permanent magnet.

\section{Proposed Passive Fault Current Limiter Model}

The basic idea of the passive fault current limiter proposed in [9] is to use a magnetic core with a permanent magnet inserted in the gap between the core halves to provide a magnetic offset. The physical configuration for an E type core is shown in Figure 2.

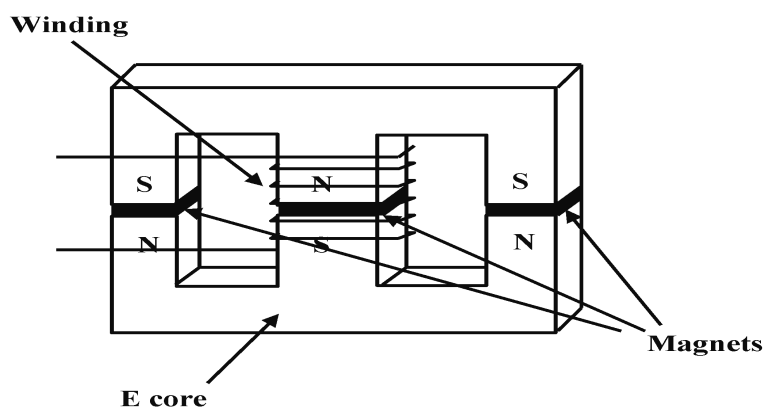

Figure 2: Passive FCL Physical Configuration

To model this type of component using a behavioral model requires three main elements, the non-linear magnetic core, the winding and the magnets. We can simplify this physical model into the three primary elements as shown in Figure 3.

The model structure shows how we model the FCL using the electrical and magnetic domains. The advantage of this approach is that we can see in the simulation results how the flux and MMF behavior compares with the measured FCL directly. The non-linear core model is based on the well-known Jiles-Atherton approach $[12,13,14]$ and the winding model is again the same as has been used pre-

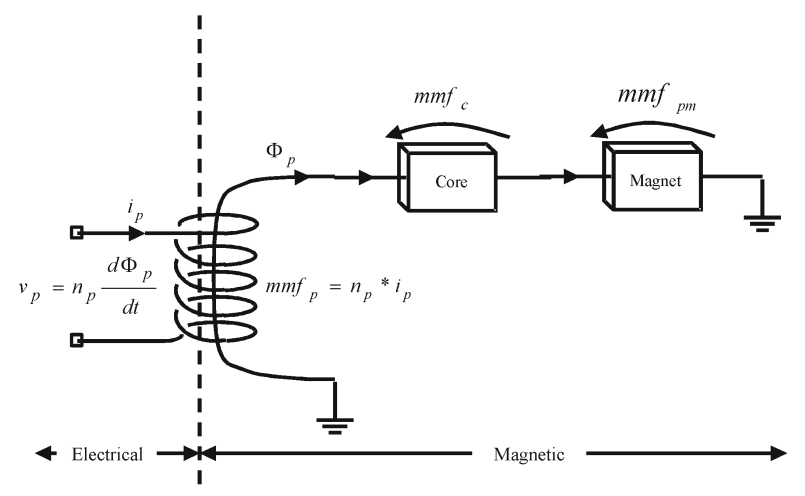

Figure 3: Proposed Model Structure

viously in this field $[17,18,19,20,21]$. Modeling permanent magnets can be achieved using a simple magnetic offset model of the form shown in Figure 4.

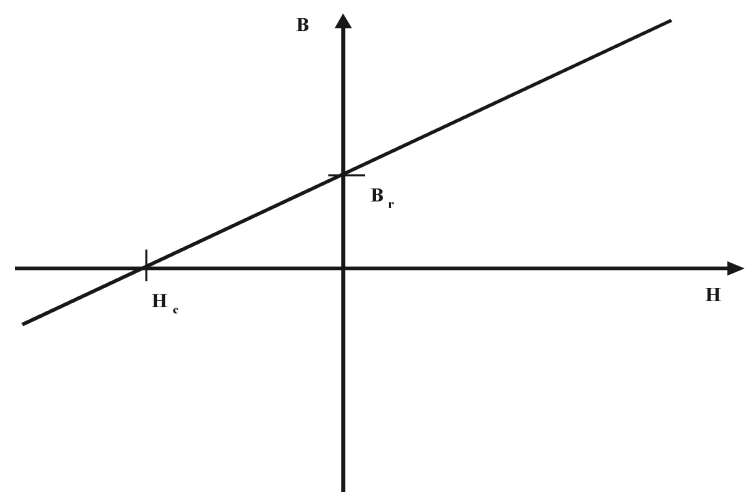

Figure 4: Permanent Magnet model behavior

Using this method a simple linear reluctance model, with a D.C. MMF source of $-\mathrm{Hc}$ will give the required behavior. The model used in this work is given in Figure 5, showing how the effective magnetic field strength $\mathrm{He}$, is calculated internally to the model.

.SUBCKT PMAGNET 12 PARAMS: UR $=1000$

$+\mathrm{HC}=-100$ AREA $=10 \mathrm{e}-6$ LEN $=10 \mathrm{e}-3$

E1 B 0 VALUE $=\{\mathrm{V}(\mathrm{HE}) *(12.8 \mathrm{e}-7 * \mathrm{UR})\}$

E2 MMF 0 VALUE $=\{\mathrm{V}(1,2)\}$

E3 H 0 VALUE $=\{\mathrm{V}(\mathrm{MMF}) / \mathrm{LEN}\}$

E4 FLUX 0 VALUE $=\{\mathrm{V}(\mathrm{B}) * \mathrm{AREA}\}$

E5 HE 0 VALUE $=\{\mathrm{V}(\mathrm{H})-\mathrm{HC}\}$

G1 112 FLUX 001

.ENDS

Figure 5: Linear Permanent Magnet Model

Of course, permanent magnets have a non-linear behavior just like any other magnetic material and if necessary a more accurate representation of the demagnetization curve of the material can be implemented as shown in Figure 6.

In order to characterize this behaviour, a Langevin 


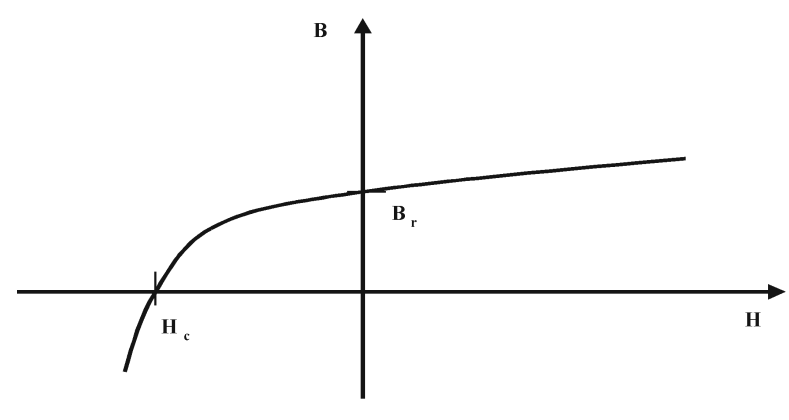

Figure 6: Permanent Magnet Demagnetization

function was used (also used in the Jiles Atherton Model $[12,13,14]$ ), with an $\mathrm{H}$ axis offset added to provide this behaviour. The listing of this model is given in Figure 7.

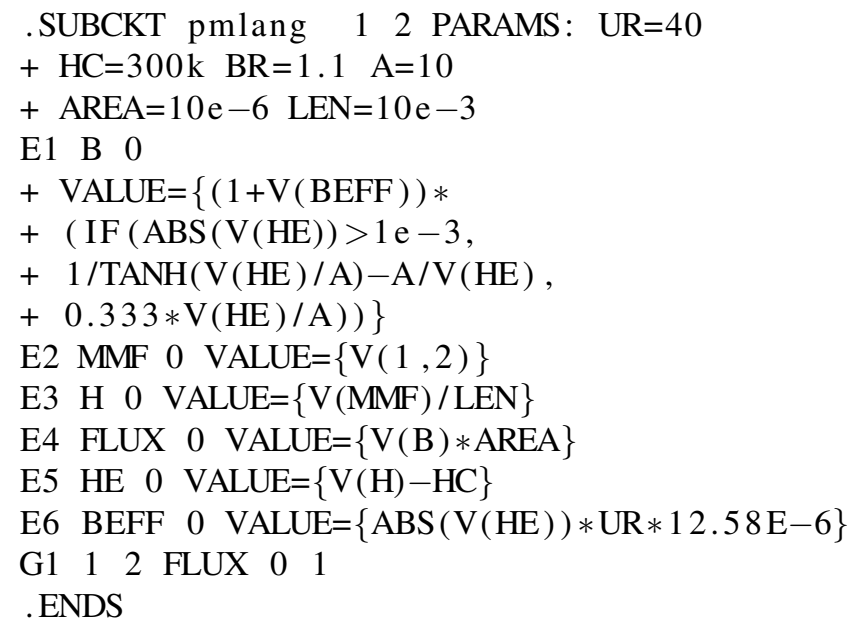

Figure 7: Permanent Magnet Model based on the Langevin Function

These approaches are satisfactory for most cases, and were used successfully in this project, but in fact the magnet behavior shown thus far is just a small section of an overall BH hysteresis loop which can be modeled using standard models such as Jiles-Atherton. This becomes important when the effect of magnet recoil is relevant to the behavior of the device. Figure 8 illustrates this aspect of the magnet's behavior. As the magnet is demagnetized (Path $\mathrm{AB}$ ), if the applied field strength changes direction, then the magnet does not return back the same way, but traverses the $\mathrm{BH}$ loop across to the other side directly (Path $\mathrm{BC}$ and then to D). Thus the effective permeability is the same, but the flux density is much lower.

Implementing a very wide square loop of the kind shown in Figure 8 using the standard Jiles-Atherton model is not easy, as the equations are not designed for a Coercive Force of $10 \mathrm{e} 6 \mathrm{At} / \mathrm{m}$, and will potentially become unstable. To improve the robustness of the model therefore, the effective field strength was scaled in the magnet model by a large factor (e.g. 100,000 A/m) to keep the model within a sensible numerical region. Given that the small numerical

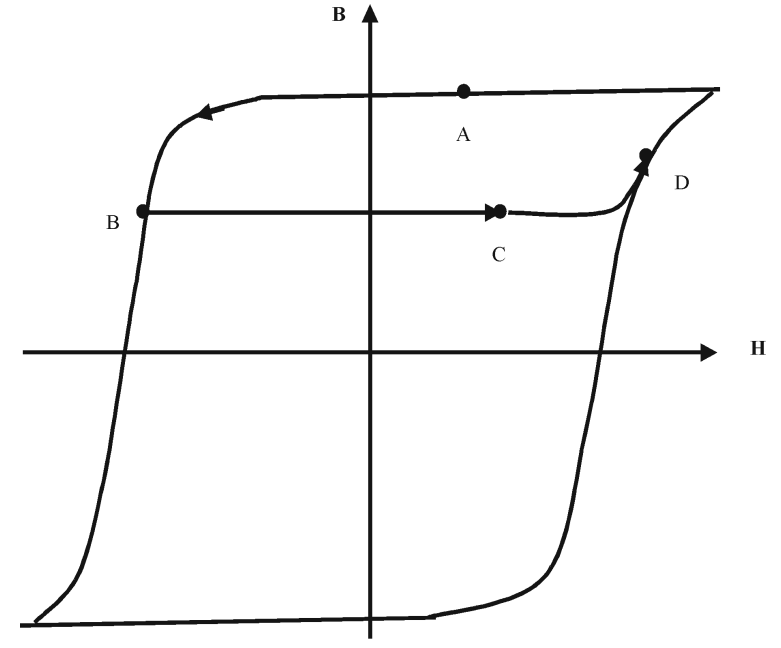

Figure 8: Illustration of Magnetic Recoil Trajectory

differences in the model can be much smaller than this, by scaling the absolute value accordingly, the numerical accuracy can be maintained in the simulator (i.e. if the relative sizes of the smallest and largest numbers in a simulation are too far apart, then this can result in truncation error of the smaller numbers).

With the range of models defined from a simple linear magnetic offset to more complex non-linear and hysteresis approaches, the application can be modeled with the required level of accuracy. As a benchmark and comparison, a single offset winding was added to the same core material to provide an alternative modeling representation. This was then compared, both with measured and simulated results, to establish the relative merits of the models used in each case.

\section{Fault Current Limiter using a Permanent Magnet offset}

In this section a practical Permanent Magnet FCL was constructed, tested and the results compared with the simulation model. Using the averaged $\mathrm{BH}$ curve and corresponding Impedance curve in Figure 9, the characteristics of the FCL can be designed.

If the nominal operating point is at $\mathrm{A}$, then it can be seen that the slope of the BH curve is low, therefore the corresponding impedance at $\mathrm{A}$ is also low. This is the normal operating condition and the FCL does not limit the current. If the current is perturbed around this point, for small values of current perturbation there will be no impact on the waveform due to the FCL. If the current perturbation increases, however, to the extent that point $\mathrm{B}$ is reached, then the slope of the $\mathrm{BH}$ curve increases as does the impedance. This is the region (B) in which the impedance of the FCL causes limiting of the current. If the current continues to rise, despite the efforts of the FCL, and continues further into region $\mathrm{C}$, the magnetic core will go back into saturation, with the result that the $\mathrm{BH}$ slope decreases, as does 

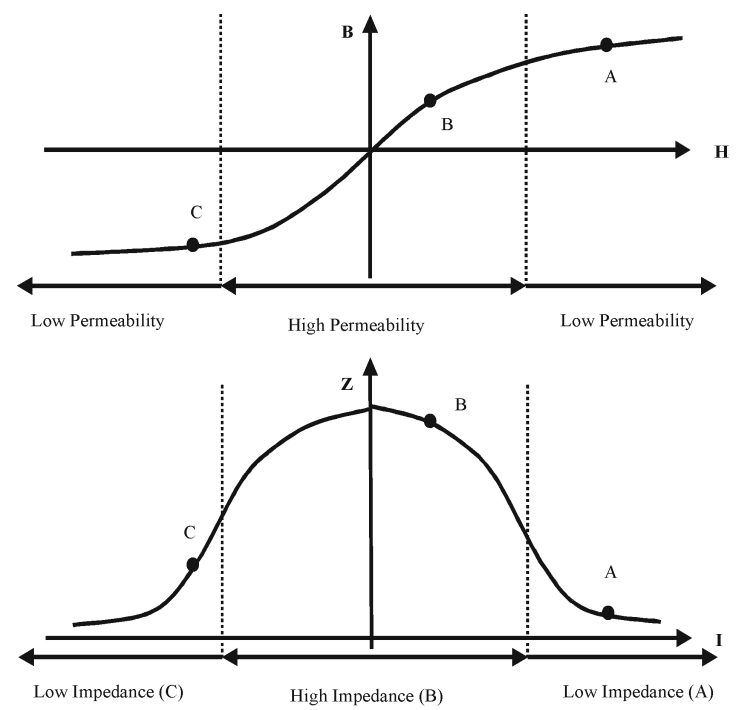

Figure 9: FCL Averaged BH \& Impedance Curves

the impedance of the FCL. It is also clear from these graphs that to obtain bi-polar current limiting, two of these devices need to be placed in series, with opposite magnetic offsets. The basic design methodology uses the classical equation for inductance given in Eq. (1).

$$
L=\frac{\mu_{0} \mu_{r} N^{2} A}{l}
$$

Where $A$ is the cross sectional area of the core, $l$ is the effective magnetic path length, $\mathrm{N}$ is the number of turns, $\mu_{0}$ is the permeability of free space, and $\mu_{r}$ is the relative permeability of the core material. When the material is in saturation (i.e. normal operation) the relative permeability is much lower, hence a lower inductance and impedance results than for the fault condition.

\section{Construction and Measurement}

The choice of a toroidal core type was made for a number of reasons. Primarily, the need to keep the modeling structure as simple as possible to reduce the probability of errors due to the complexity of the structure of say, a multiple section E core. This also allows pure analytical calculations to be carried out, estimating magnetic path lengths, and keeps the cross sectional area of the magnetic flux path constant. The choice of magnet also enables the flux path area to be the same shape and area as that of both the core and the magnet, reducing the errors introduced by fringing and leakage fluxes.

By reducing the complexity of the core/magnet combination to a toroid, the number of air gaps and magnets is also reduced, thus increasing the confidence in the model and simulation results. The prediction of the model parameters is also made simpler by this design decision. The FCL design layout used in this paper is shown in Figure 10. The core was based on a Philips 23/14/7 toroid, made of the 3F3 material. A $1 \mathrm{~mm}$ gap was cut in the toroid, as shown in Fig- ure 10 , enabling the magnet ( $1 \mathrm{~mm}$ thick) to fit exactly into the gap.

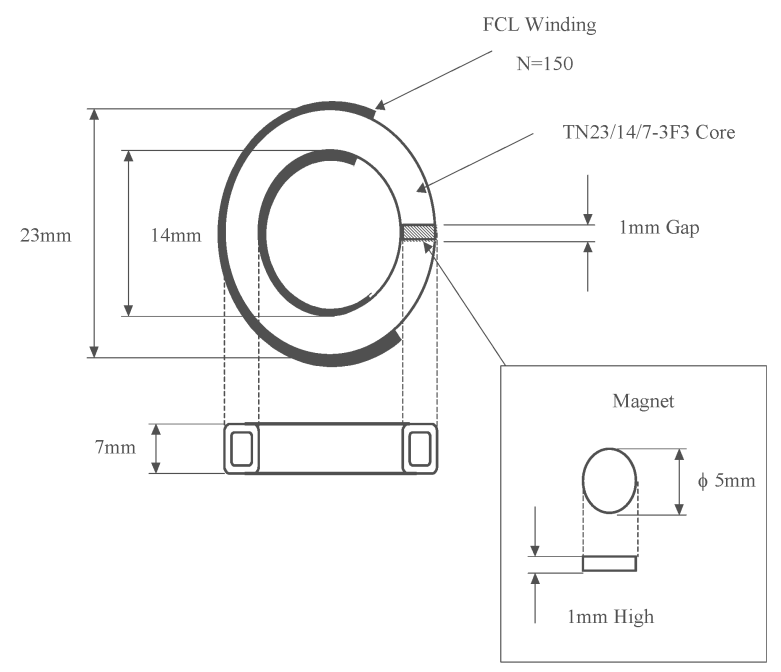

Figure 10: FCL Based on TN29/19/17.6-3F3 Toroid

The toroid was wound with 150 turns, cross sectional area of $30.9 \mathrm{~mm}^{2}$, magnetic path length $55.8 \mathrm{~mm}$. The effective inductance was measured with and without the magnet in place. The magnet used was a NdFeB permanent magnet, $5 \mathrm{~mm}$ diameter and $1 \mathrm{~mm}$ thick.

A secondary winding was wound around the outside to monitor the voltage across the toroid for the purposes of $\mathrm{BH}$ curve estimation (in all the measurement graphs, the initial Flux Density, B, has been assumed to be 0T, although in practice it will be a non-zero value. It is not possible from the voltage measurements alone to calculate any steady state initial flux conditions, unless the full start up transients are taken into the calculation). A current sense resistor was used in series with the winding to derive the current through the FCL inductor, and the line voltage from the source was also measured.

\section{Measurement Results}

To test the basic behavior of the FCL, a current sense resistor doubling as a load of $10 \Omega$ was used in series with the toroidal FCL. The secondary winding of 100 turns was used to measure the voltage and derive the flux density through the core. Using this configuration, a line voltage was applied to the FCL and Load combination, with the amplitude gradually increased until the FCL began to limit the line voltage and the frequency set to $100 \mathrm{~Hz}$. With low levels of voltage, the line current and sensed voltage across the FCL are nearly sinusoidal as shown in Figure 11. This indicates that the FCL is operating in the saturated (linear) region, and has minimal effect on the waveform shape of the line voltage. This is confirmed when the Flux Density (B) and Magnetic Field Strength $(\mathrm{H})$ in the core are calculated, the resulting BH curve is shown in Figure 12. This shows that the permeability is effectively constant.

As the line voltage increases, or the load resistance is 


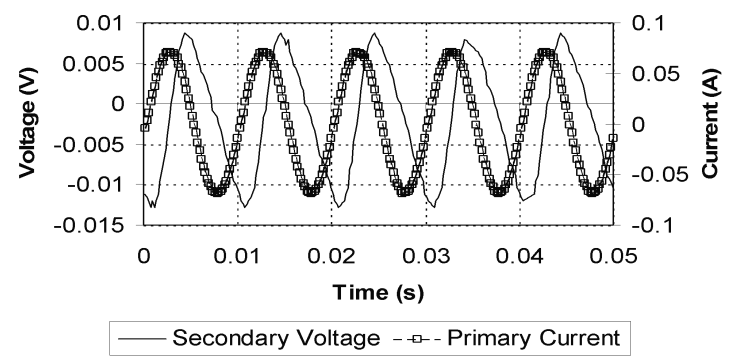

Figure 11: FCL Line Current and Secondary Voltage

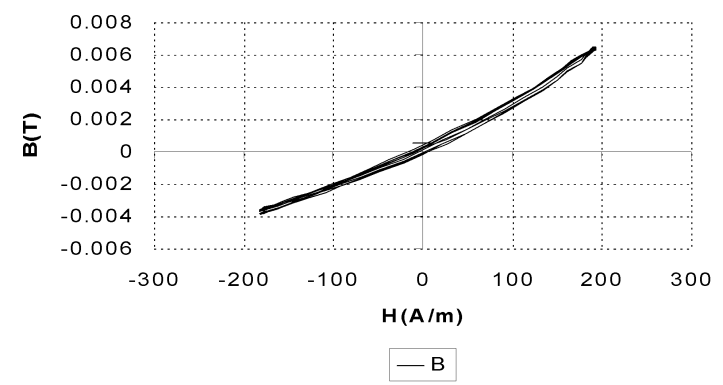

Figure 12: BH Curve derived from FCL operation under nominal load conditions

reduced to simulate the effect of a fault condition, the deviation of the current will push the core material out of saturation and cause a distortion of the line current, and provide effective fault current limiting. Using the same test configuration, the line voltage was increased, with the resulting line current and sensed voltage waveforms obtained in Figure 13.

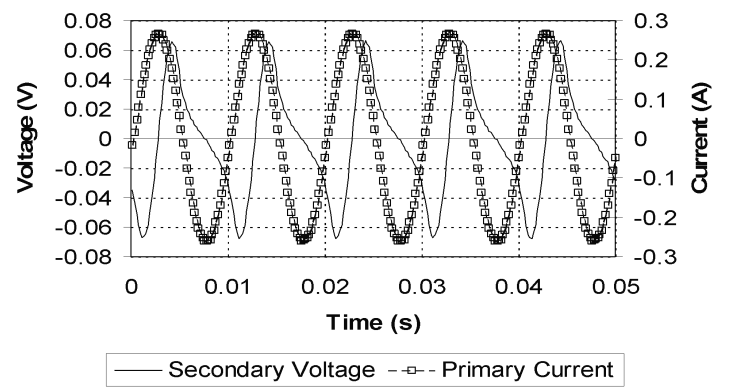

Figure 13: FCL Sensed Line Current and Secondary Monitor Voltage Waveforms

As can be seen from Figure 13, during the positive half cycle of the line current, there is a phase shift of the voltage, but no significant distortion, however, during the negative half cycle, the sensed voltage is limited and is due to the magnetic material coming out of saturation. This is clearly seen in Figure 14, which shows the BH curve of the magnetic material under the same load and line conditions.

It is noticeable that the graph is no longer linear, and that the area of the $\mathrm{BH}$ curve is beginning to become vis-

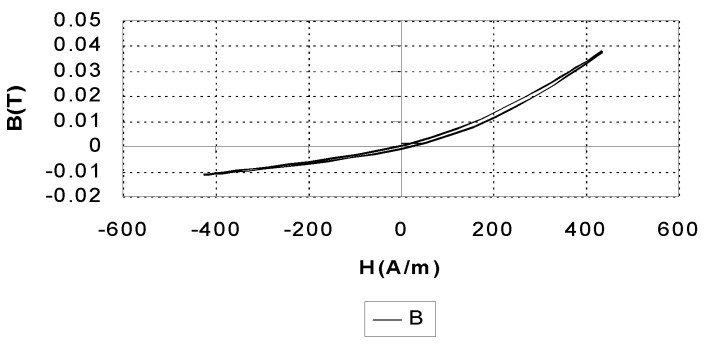

Figure 14: BH Curve for FCL under increased Line Voltage Conditions

ible, i.e. the core is moving into hysteresis. The load was then reduced to $0.5 \Omega$, resulting in a simulation of a fault condition, such as a short across the line. When the $\mathrm{BH}$ curve is plotted under these conditions, the hysteresis behavior becomes pronounced as shown in Figure 15. The frequency of the line voltage was reduced to $5 \mathrm{~Hz}$ to reduce eddy currents in the magnet (and the resulting self-heating).

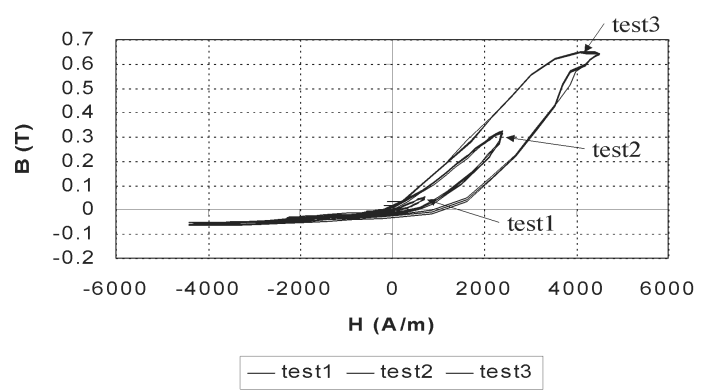

Figure 15: FCL BH Curve under Fault Conditions (Load = $0.5 \mathrm{Ohm})$

Figure 15 illustrates one of the reasons why using a simple PWL model of the magnetic core is not adequate for predicting the behavior of a practical FCL. The hysteresis behavior defines the loss in the component, and also directly influences the voltage and current waveforms in the limiter. By implementing a model with this behavior, a potentially highly accurate model may result.

\section{Comparison of Simulated and Measured Results}

In order to model the fault current limiter used to obtain the measurements, the structure previously described in Figure 3 was used. A non-linear model of the magnetic core material ( $3 \mathrm{~F} 3$ ) was used, characterized using the techniques described previously in previous work in modeling the behavior of magnetic materials. This was then combined with a simple linear model of the permanent magnet in the first instance and the measured line voltage was used as the stimulus to allow a direct comparison with the measured results.

Figure 16 shows the comparison between the the measured and simulated flux densities (Bmeas and Bsim respec- 
tively) plotted against time. An offset was added to the derived, measured flux density (Bmeas) for easy comparison with the simulated equivalent.

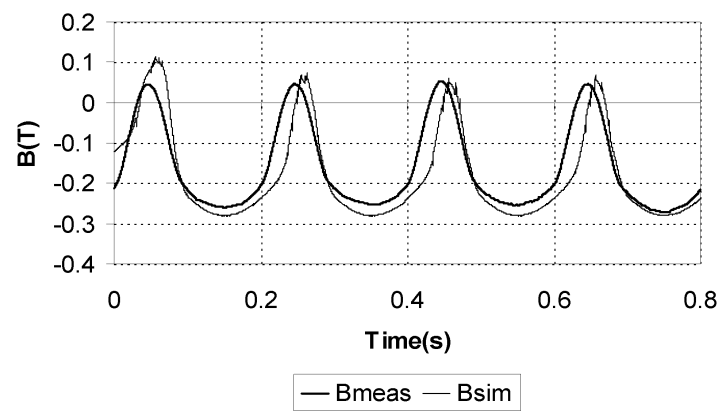

Figure 16: Measured and Simulated Flux Densities

It is clear that despite accurate material modeling of the $3 \mathrm{~F} 3$ material, and a simple configuration, there were some problems with the results. Looking at the $\mathrm{BH}$ curves, it is clear that the simulated $\mathrm{BH}$ curve is fatter and steeper than the measured equivalent. This seems to indicate an aspect of the FCL behavior not considered in the model. When the time domain plot in Figure 16 is observed, it appears that there also may be a phase shift introduced that is not included in the model.

The material model characterization software [21] was modified to allow the characterization of the Jiles-Atherton model in combination with a permanent magnet offset. Tests using this approach improve the matching of the $\mathrm{BH}$ curve, but the phase difference in the time domain remains. This difference needed a frequency dependent addition to the model which is discussed in the next section of this paper.

\section{Magnet Model Modification}

The magnet model was modified using the model structure shown in Figure 17. This extends the basic linear model of a reluctance in series with a magnetic field strength $(\mathrm{H})$ offset in the magnetic domain, by including a magnetic capacitor element across the reluctance of the magnet. This combination gives a dependence of the magnetic reluctance with frequency, that can provide a phase shift commensurate with the measured data.

Using this modified model, with a reluctance of $100 \mathrm{k}$, and a magnetic capacitance of $0.5 \mu \mathrm{F}$, the phase shift error was corrected as shown in figure 18. These values were obtained by optimizing the reluctance and capacitance values using iterative simulations in SPICE. Using this modification is clearly non-physical, as a magnetic 'capacitance' is a pure energy source, but it is a simple method of improving the phase behavior of the model compared to the measured results. It is a weakness of existing magnet models that could be the subject of future work.

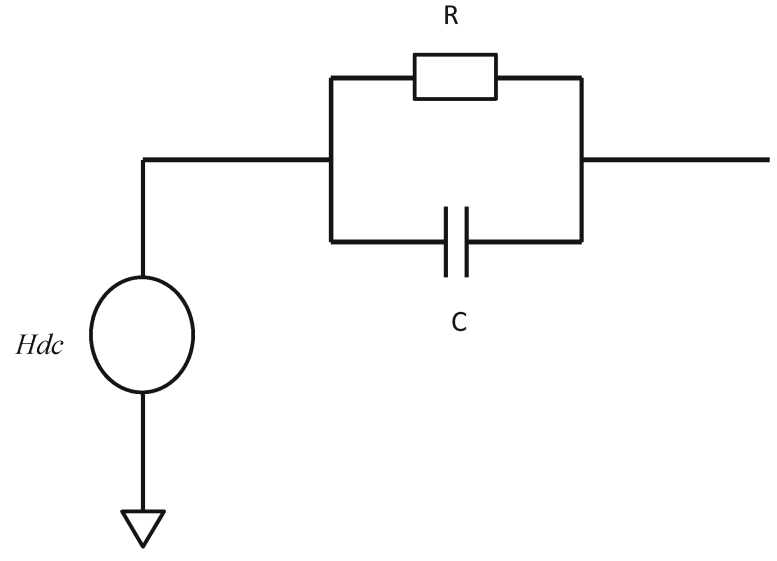

Figure 17: Modified Magnet Model with Frequency Dependent Reluctance

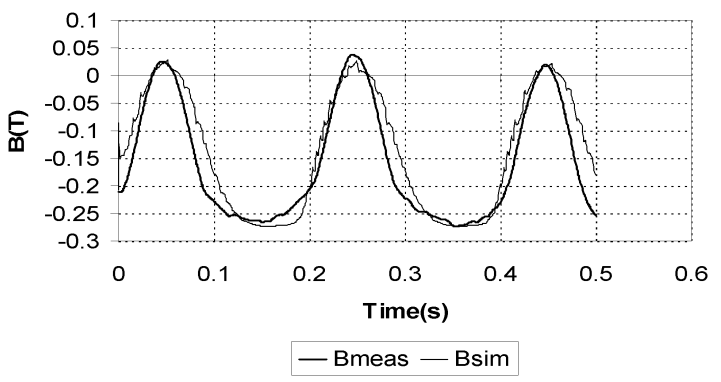

Figure 18: Flux Density of the FCL with corrected phase shift

\section{Conclusions}

The results obtained of the behaviour of the fault current limiting inductor using a non-linear Jiles-Atherton core model have shown that by using a true hysteresis model, the BH trajectory of the FCL can be predicted using simulation and matched closely to the physical device under fault and nominal conditions. With reference to the previous work in [9] and [10], one of the deficiencies with the models used was the lack of true hysteresis behavior, leading to discrepancies between the simulated and measured results, showing up as asymmetry in the line voltage and current waveforms. The authors did not explain these discrepancies, but it is clear from BH curves in the tests carried out that such asymmetry could result from hysteresis in the magnetic core material. By using a mixed-technology approach to modeling the magnetic elements in the FCL, this aspect of the component can be modeled more accurately, and allows a higher level of confidence in the design of the FCL.

\section{Potential for Further Work}

The modeling effort has primarily concentrated on the magnetic core material behavior, while using a fairly simple linear magnet model. The FCL design is primarily for use at low frequencies $(; 100 \mathrm{~Hz})$, and modeling of eddy currents 
in the magnet has been neglected. Despite these omissions, the results have had adequate accuracy for this application. Experimental work previously carried out at higher frequencies, however, has highlighted the potential problems of eddy currents in the magnet, to the extent of thermal demagnetization occurring. Useful future work could therefore be carried out in this area, extending the magnet model to include hysteresis, and using eddy current models.

\section{References}

[1] "Fault Current Limiting - present situation and future needs", CIGRE 23-79-(WG-04)-06, 1979

[2] King EF, Chikhani AY, Hackam R, Salama MMA, A Microprocessor-Controlled Variable Impedance Adaptive Fault Current Limiter, IEEE Transactions on Power Delivery, Vol. 5, No. 4, pp1830-1838, Oct. 1990

[3] Ibrahim ES, Electromagnetic fault current limiter, Electric Power Systems Research, Vol. 42, No. 3, pp189-194, Sep. 1997

[4] Inaba T , Performance of a Fault-Current Current Limiter With a New Type of Direct Cooled Tubular Element, IEEE Transactions on Power Apparatus and Systems, Vol. 103, No. 7, pp1888-1894, 1984

[5] Karady GG, Principles of Fault Current Limitation by a resonant LC circuit, IEE Proceedings-C Generation Transmission and Distribution, Vol. 139, No. 1, pp16, Jan. 1992

[6] Ishigohka T, Sasaki N, Fundamental Test of New DC Superconducting Fault Current Limiter, IEEE Transactions on Magnetics, Vol. 27, No. 2, pp2341-2344, Mar. 1991

[7] Ito D, Yoneda ES, Tsurunaga K, Tada T, Hara T, Ohkuma T, Yamamoto T, 6.6 KV/1.5 KA-Class Superconducting Fault Current Limiter Development, IEEE Transactions on Magnetics, Vol. 28, No. 1, pp438-441, Jan. 1992

[8] Ito D, Tsurunaga K, Yoneda ES, Sugiyama Y, Hara T, Okaniwa K, Hoshino H, Yamamoto T, Superconducting Fault Current Limiter Development, IEEE Transactions on Magnetics, Vol. 27, No. 2, pp2345-2348, Mar. 1991

[9] Mukhopadhyay, S. C.; Dawson, F. P.; Iwahara, M.; Yamada, S., Analysis, design and experimental results for a passive current limiting device, IEE Proceedings: Electric Power Applications, Vol. 146, No. 3, (1999), pp309-316

[10] Dawson FP, Yamada S, Iwahara M, Experimental Results for a 2-Material Passive Di/Dt Limiter, IEEE Transactions on Magnetics, Vol. 31, No. 6, pp37343736, Nov. 1995
[11] Iwahara M, Miyazawa E, A Numerical Method for calculation of Electromagnetic Circuits using the tableau approach, IEEE Transactions on Magnetics, Vol. 19, No. 6, pp2457-2460, 1983

[12] DC Jiles, DL Atherton, Theory of Ferromagnetic Hysteresis (invited), Journal of Applied Physics, Vol. 55 , No 6, Mar 1984, 2115-2120

[13] D.C. Jiles, D.L. Atherton, Theory of Ferromagnetic Hysteresis, Journal of Magnetism and Magnetic Materials, 61, 1986, 48-60

[14] D.C. Jiles, D.L. Atherton, "Ferromagnetic Hysteresis", IEEE Transactions on Magnetics, Vol 19, No. 5, Sep 1983, pp2183-2185

[15] Rozenshtein, V.; Friedman, A.; Wolfus, Y.; Kopansky, F.; Perel, E.; Yeshurun, Y.; Bar-Haim, Z.; Ron, Z.; Harel, E.; Pundak, N., "Saturated Cores FCLA New Approach," IEEE Transactions on Applied Superconductivity, vol.17, no.2, pp.1756,1759, June 2007

[16] Hall, J.; Cheer, A., "Fault current limiter surge protection device for the power grid based upon zero power consumption ceramic ferrite permanent magnets," 22nd International Conference and Exhibition on Electricity Distribution (CIRED 2013), , vol., no., pp.1,4, 10-13 June 2013

[17] P.R. Wilson, J.N Ross, A.D. Brown, Predicting total harmonic distortion in asymmetric digital subscriber line transformers by simulation, IEEE Transactions on Magnetics, Vol. 40 , Issue: 3 , 2004, pp. 15421549

[18] P.R. Wilson, J.N Ross, A.D. Brown, Modeling frequency-dependent losses in ferrite cores, IEEE Transactions on Magnetics , Vol. 40 , No. 3 , 2004, pp. 15371541

[19] P.R. Wilson, J.N Ross, A.D. Brown, "Simulation of Magnetic Component Models in Electric Circuits including Dynamic Thermal Effects", IEEE Transactions on Power Electronics, Vol. 17, No. 1, 2002, pp. $55-65$

[20] Al-Junaid, H., Kazmierski, T., Wilson, P. and Baranowski, J., Timeless Discretization of the Magnetization Slope in Modeling of Ferromagnetic Hysteresis, IEEE Transactions on Computer-Aided Design of Integrated Circuits and Systems, Vol. 25, No. 12, pp2757-2764, 2006.

[21] P.R. Wilson, J.N Ross, A.D. Brown, Magnetic Material Model Characterization and Optimization Software, IEEE Transactions on Magnetics, Vol. 38, No. 2, Part 1, 2002, pp. 1049-1052 